Supporting Information

\title{
Photoelectric Logic and In Situ Memory Transistors with Stepped Floating Gates of Perovskite Quantum Dots
}

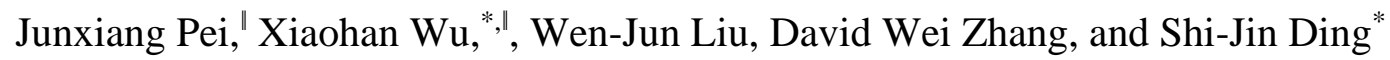

Figure S1. Evaporation of $\mathrm{CsPbBr}_{3}$ PQDs at different substrate temperatures. a-c) SEM images and d) PL spectra of the $\mathrm{CsPbBr} 3$ PQDs deposited at different substrate temperatures (nominal thickness: $20 \mathrm{~nm}$ ).
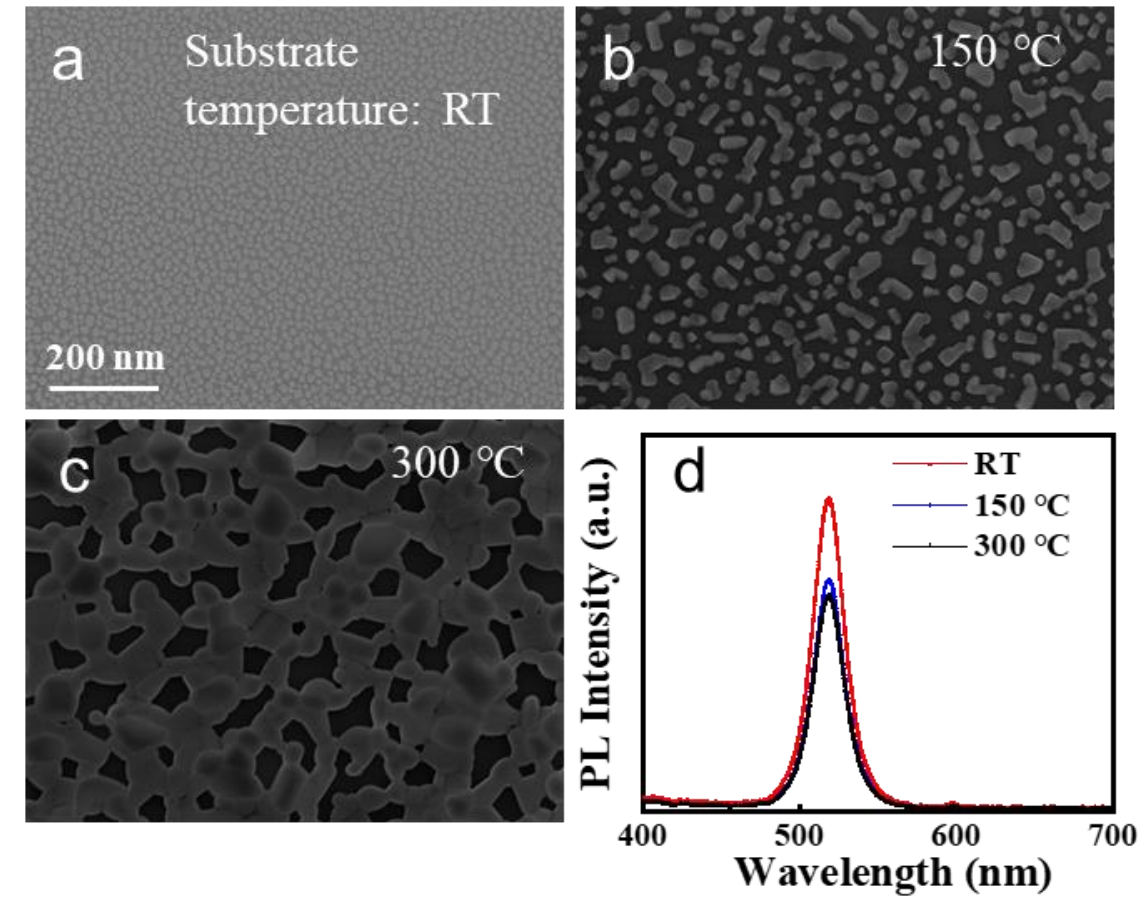
Figure S2. Fabrication of $\mathrm{CsPbBr}_{3}$ PQDs with different annealing temperatures. a-d) SEM images and e) PL spectra of the as-deposited $\mathrm{CsPbBr}_{3} \mathrm{PQDs}$ and the PQDs annealed at different temperatures (Substrate temperature: RT, nominal thickness: $20 \mathrm{~nm}$, and annealing time: 30 $\min )$.
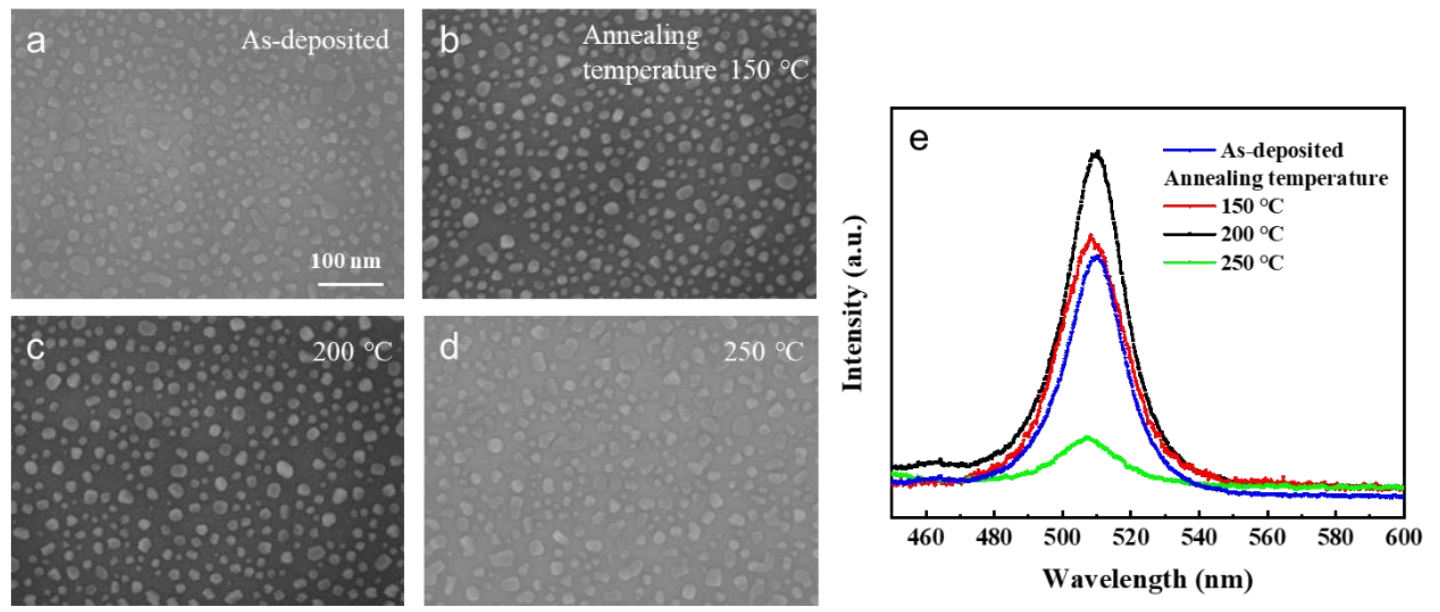

Figure S3. Fabrication of $\mathrm{CsPbBr}_{3}$ PQDs with different annealing times. a) SEM images and b) PL spectra of the as-deposited $\mathrm{CsPbBr}_{3}$ PQDs and the PQDs annealed for 60 min (Substrate temperature: RT, nominal thickness: $20 \mathrm{~nm}$, and annealing temperature: $200{ }^{\circ} \mathrm{C}$ ).

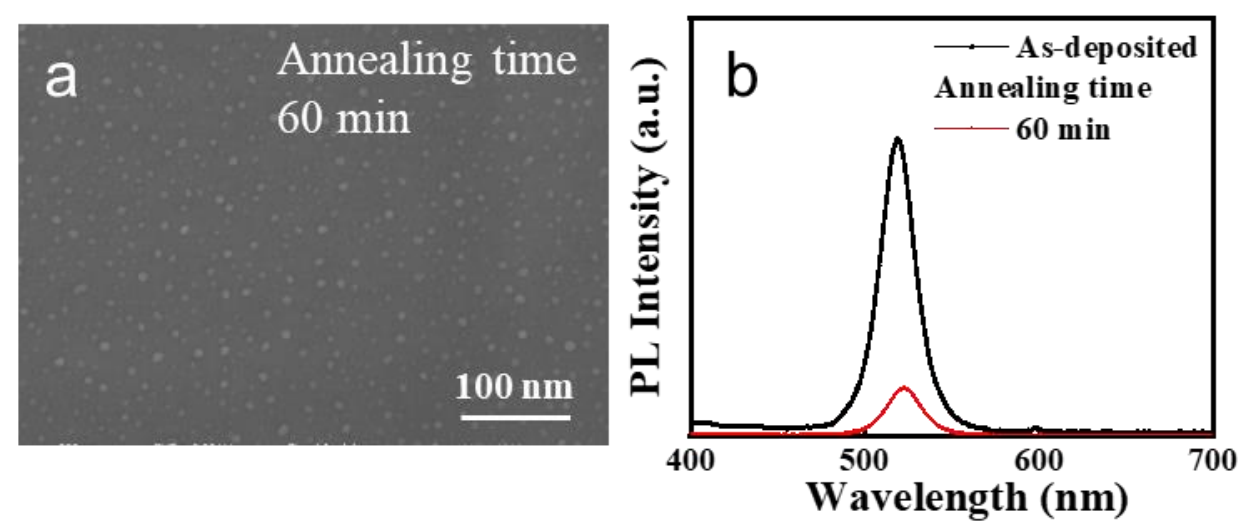


Figure S4. Scheme of the fabrication processes for the stepped PQDs-FGs transistor.

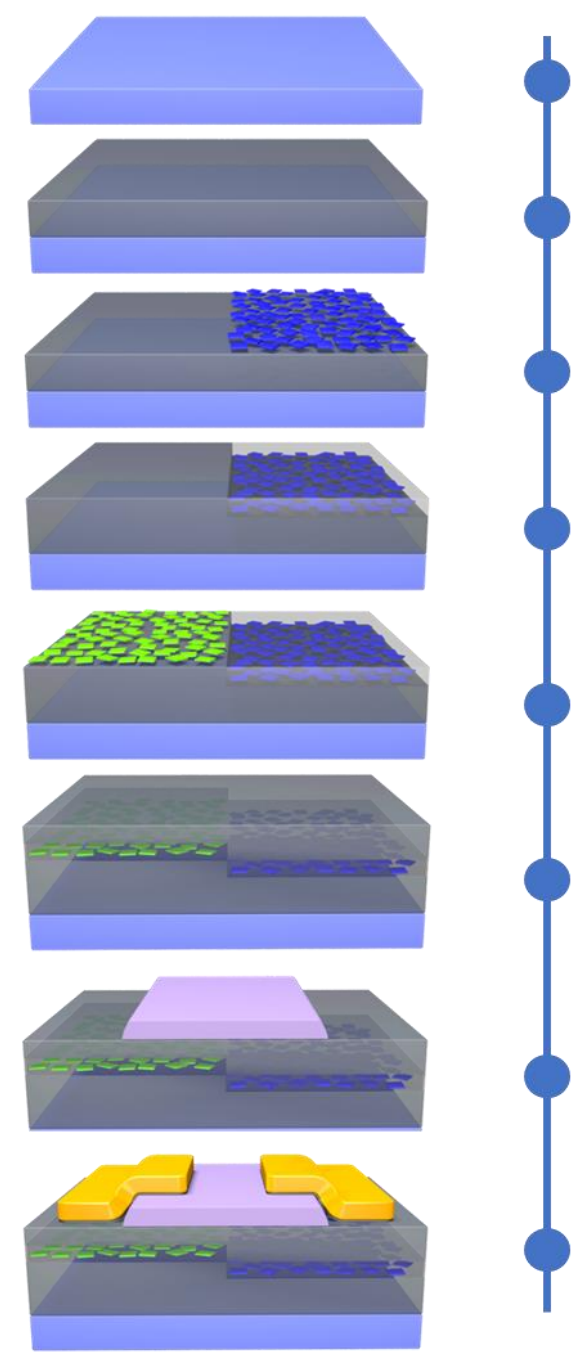

Si Substrate cleaned by RCA process

$\mathrm{Al}_{2} \mathrm{O}_{3}$ blocking layer deposited by $\mathrm{ALD}$

$\mathrm{CsPbCl}_{2} \mathrm{Br}$ half-FG deposited by thermal evaporation

$\mathrm{Al}_{2} \mathrm{O}_{3}$ thin film deposited by $\mathrm{ALD}$

$\mathrm{CsPbBr}_{3}$ half-FG deposited by thermal evaporation

$\mathrm{Al}_{2} \mathrm{O}_{3}$ tunneling layer deposited by $\mathrm{ALD}$

a-IGZO channel layer deposited by PVD

Source/Drain electrode deposited by thermal evaporation 
Figure S5. Dynamic switching characteristics of the stepped PQDs-FGs transistors.

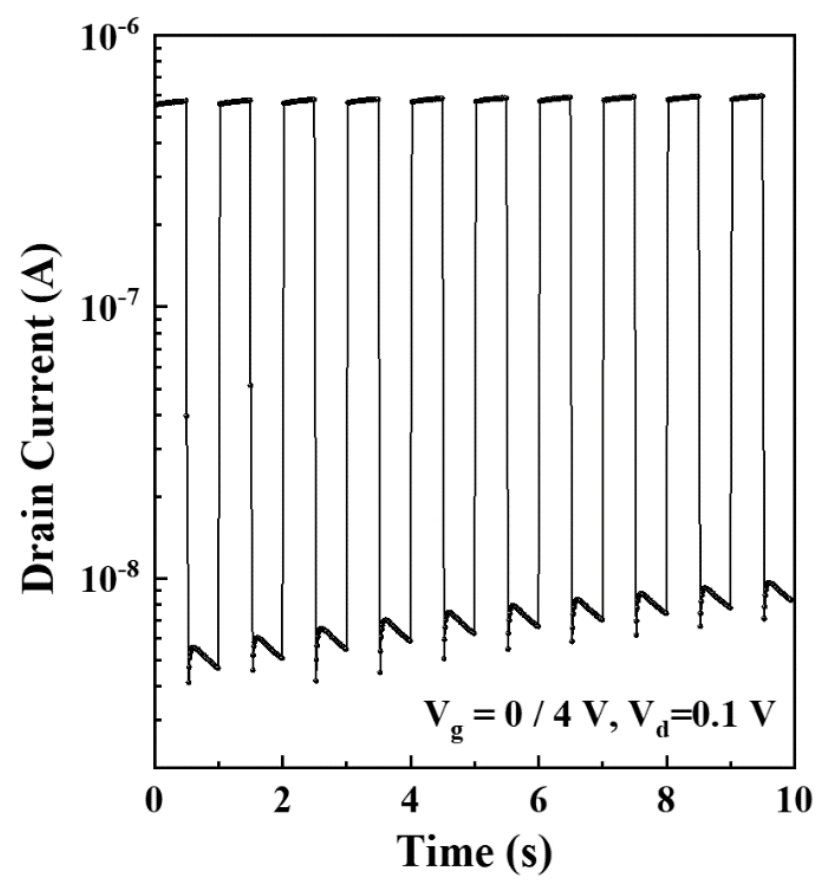

Figure S6. Transfer curves of different stepped PQDs-FGs transistors.

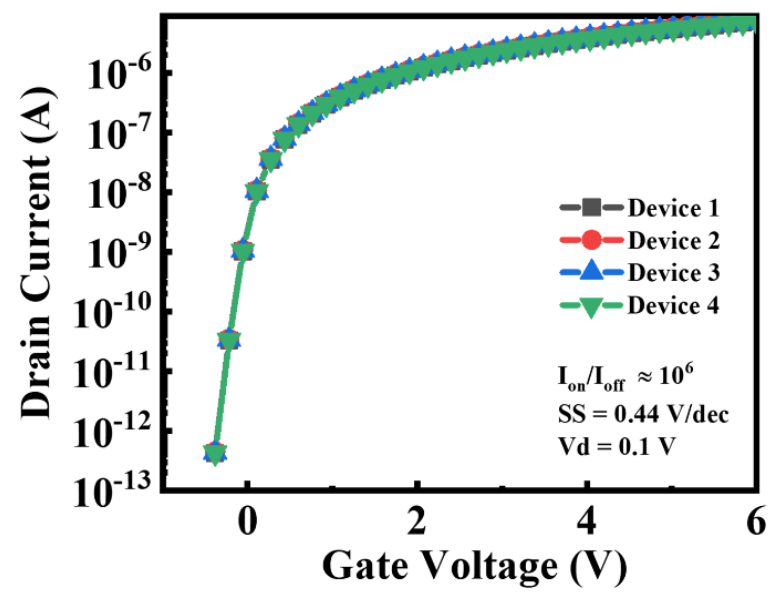


Figure S7. Energy band diagrams of the stepped PQDs-FGs transistor writing with a) relatively low positive voltage in the dark, b) under rather long and c) short wavelength light, and d) relatively high positive voltage in the dark, e) under rather long and f) short wavelength light.

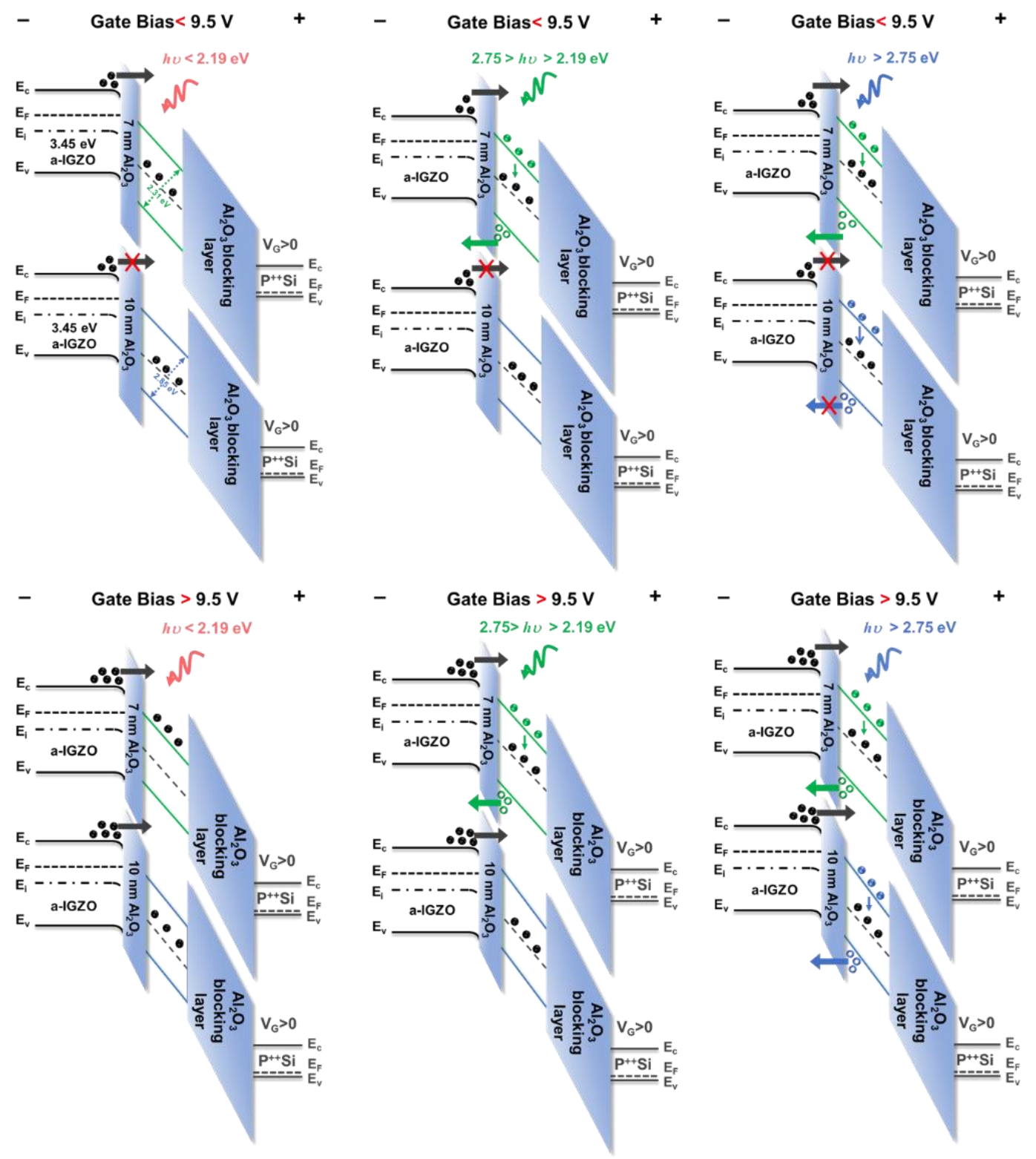


Figure S8. Transfer curves of the stepped PQDs-FGs transistor in the four different memory states.

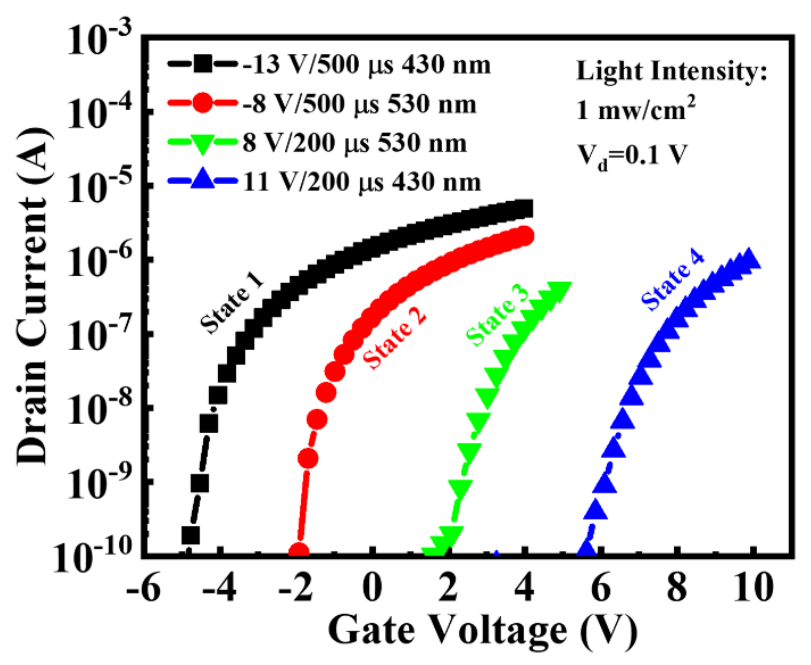

Figure S9. Realization of AND gate on the stepped PQDs-FGs transistor with another group of input signals: transfer curves of the device written after IN 1 with relatively low voltages and IN 2 with rather long wavelengths.

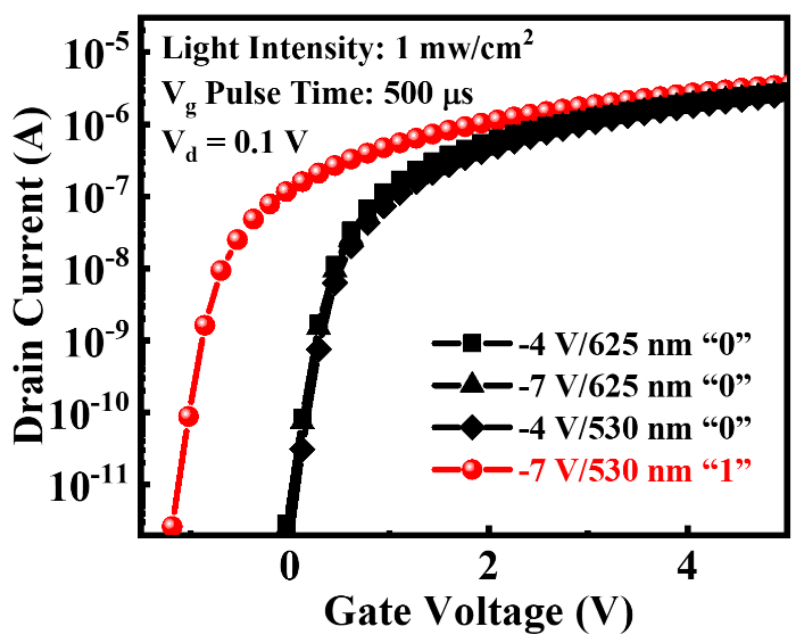


Figure S10. Illustration of realizing logic OR by designing the stepped PQDs-FGs transistor.

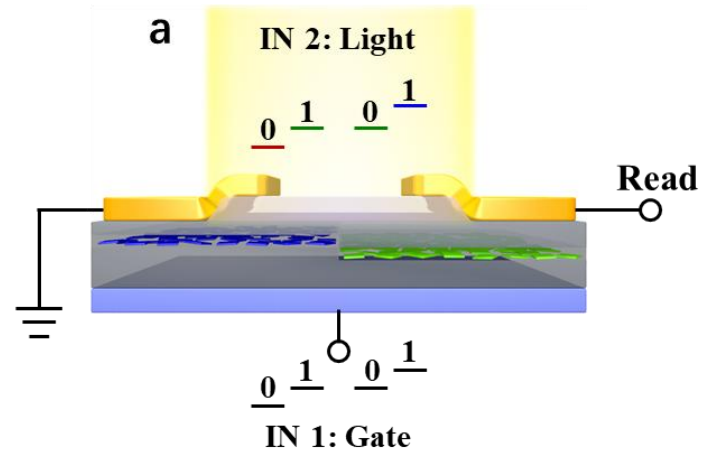

\begin{tabular}{|c|c|c|c|c|}
\hline \multirow[t]{2}{*}{ b } & \multirow{2}{*}{$\begin{array}{c}\text { Logic OR } \\
+ \\
\text { Memory }\end{array}$} & $\begin{array}{l}\text { IN } 1 \\
\text { Gate }\end{array}$ & $\begin{array}{l}\text { IN } 2 \\
\text { Light }\end{array}$ & $\begin{array}{c}\text { Output } \\
\mathbf{V}_{\text {th }}\end{array}$ \\
\hline & & $\mathbf{0}(\mathrm{V}>-9.5 \mathrm{~V})$ & $\boldsymbol{0}(\lambda>430 \mathrm{~nm})$ & $\mathbf{0}$ (low $\mathrm{V}_{\text {th }}$ ) \\
\hline & \multirow[b]{2}{*}{$\&$} & $\mathbf{0}(\mathrm{V}>-9.5 \mathrm{~V})$ & $\mathbf{1}(\lambda \leq 430 \mathrm{~nm})$ & $\mathbf{1}\left(\right.$ high $\left.\mathrm{V}_{\text {th }}\right)$ \\
\hline & & $\mathbf{1}(\mathrm{V} \leq-9.5 \mathrm{~V})$ & $\boldsymbol{0}(\lambda>430 \mathrm{~nm})$ & 1 (high $\left.V_{\text {th }}\right)$ \\
\hline & Stc & $\mathbf{1}(\mathrm{V} \leq-9.5 \mathrm{~V})$ & $\mathbf{1}(\lambda \leq 550 \mathrm{~nm})$ & $\mathbf{1}\left(\right.$ high $\left.\mathrm{V}_{\mathrm{th}}\right)$ \\
\hline
\end{tabular}

Table S1. Performance comparison between previously reported photoelectronic memories and the stepped PQDs-FGs transistor.

\begin{tabular}{|c|c|c|c|c|c|}
\hline $\begin{array}{c}\text { Device structure } \\
\text { Channel/Tunneling } \\
\text { /FG }\end{array}$ & $\begin{array}{c}\text { Writing/ } \\
\text { erasing } \\
\text { Voltage/Time }\end{array}$ & $\begin{array}{c}\text { Light } \\
\text { Intensity } \\
\left(\mathrm{mw} / \mathrm{cm}^{2}\right)\end{array}$ & $\begin{array}{c}\text { Demonstrated } \\
\text { Retention } \\
\text { Time }(\mathrm{s})\end{array}$ & $\begin{array}{c}\text { Demonstrated } \\
\text { Endurance } \\
\text { (Cycles) }\end{array}$ & Ref. \\
\hline Pentacene/PS/C60 & $70 \mathrm{~V} / 30 \mathrm{~s}$ & 0.5 & $10^{4}$ & $10^{2}$ & 1 \\
\hline DNTT/ / $\mathrm{C}_{n}-\mathrm{DNTT}$ & $60 \mathrm{~V} / 2 \mathrm{~s}$ & 5 & $10^{4}$ & $10^{2}$ & 2 \\
\hline C60/PV3D3/C70 & $40 \mathrm{~V} / 0.4 \mathrm{~s}$ & 3.76 & $10^{2}$ & 50 & 3 \\
\hline $\begin{array}{c}\mathrm{MoS}_{2} / \mathrm{Al}_{2} \mathrm{O}_{3} \\
/ \mathrm{Graphene}\end{array}$ & $80 \mathrm{~V} / 1 \mathrm{~s}$ & $0.001-5$ & $10^{4}$ & $10^{2}$ & 4 \\
\hline $\begin{array}{l}\mathrm{PtS} / \mathrm{h}-\mathrm{BN} \\
/ \text { Graphene }\end{array}$ & $30 \mathrm{~V} / 1 \mathrm{~s}$ & $10-40$ & - & $10^{3}$ & 5 \\
\hline $\begin{array}{l}\mathrm{SnS}_{2} / \mathrm{h}-\mathrm{BN} \\
\text { /Graphene }\end{array}$ & $10-40 \mathrm{~V} / 1 \mathrm{~s}$ & $\begin{array}{c}0.32-3.2 \\
n W\end{array}$ & $10^{3}$ & $10^{2}$ & 6 \\
\hline $\begin{array}{c}\mathrm{MoS}_{2} / \mathrm{cPVP} \\
/ \mathrm{Au} \text { NPs }\end{array}$ & $100 \mathrm{~V} / 1 \mathrm{~s}$ & $0.001-1$ & $10^{4}$ & $10^{2}$ & 7 \\
\hline $\begin{array}{c}\mathrm{IGZO} / \mathrm{Al}_{2} \mathrm{O}_{3} \\
/ \mathrm{CsPbX} \mathrm{X}_{3} \\
\end{array}$ & $-7 \mathrm{~V} / 500 \mu \mathrm{s}$ & 1 & $10^{4}$ & $10^{3}$ & $\begin{array}{l}\text { This } \\
\text { Work }\end{array}$ \\
\hline
\end{tabular}




\section{Reference}

1. Jeong, Y. J.; Yun, D. J.; Kim, S. H.; Jang, J.; Park, C. E. ACS Appl Mater Interfaces 2017, 9, (13), 11759-11769.

2. Li, G. S.; Hung, C. C.; Chiang, Y. C.; Lin, Y. C.; Yang, Y. F.; Yang, W. C.; Yen, H. C.; Chen, C. K.; Hsu, L. C.; Chen, W. C. Advanced Electronic Materials 2021, 2101123.

3. Kim, M.; Seong, H.; Lee, S.; Kwon, H.; Im, S. G.; Moon, H.; Yoo, S. Sci Rep 2016, 6, 30536.

4. Zhao, J.; Wei, Z.; Yang, X.; Zhang, G.; Wang, Z. Nano Energy 2021, 82, 105692.

5. Chen, Y.; Yu, J.; Zhuge, F.; He, Y.; Zhang, Q.; Yu, S.; Liu, K.; Li, L.; Ma, Y.; Zhai, T. Materials Horizons 2020, 7, (5), 1331-1340.

6. Gao, F.; Zhang, X.; Tan, B.; Zhang, S.; Zhang, J.; Jia, D.; Zhou, Y.; Hu, P. Small 2021, 17, (45), e2104459.

7. Lee, D.; Hwang, E.; Lee, Y.; Choi, Y.; Kim, J. S.; Lee, S.; Cho, J. H. Adv Mater 2016, 28, (41), 9196-9202. 